\title{
External Occulter Operations Requirements
}

\author{
Authors: $\underline{\text { M Kochte }}^{1}$, A.B. Schultz ${ }^{1}$, I.J.E. Jordan ${ }^{1}$, D. Fraquelli ${ }^{1}$, H.M. Hart ${ }^{2}$, J. Leitner ${ }^{3}$, R.D. \\ Burns $^{3}$ \\ ${ }^{1}$ Computer Sciences Corporation, STScl, Baltimore, MD, 21218, USA \\ ${ }^{2}$ Computer Sciences Corporation, Johns Hopkins University/FUSE, Baltimore, MD, 21218, USA \\ ${ }^{3}$ Goddard Space Flight Center/NASA, Greenbelt, MD, 20771
}

\begin{abstract}
This paper will discuss the system and mission requirements needed for a planet-finding mission consisting of two spacecraft - a space telescope platform and an external occulter vehicle. This two-vehicle configuration may be operated with only modest enhancements to existing space operations systems. These requirements will encompass conventional, new, and unique spacecraft operations domains. Topics this paper will explore include inter-spacecraft operations and science and mission support.
\end{abstract}

\section{Introduction}

An external, free-flying occulter [1] is a spacecraft designed to fly thousands, even tens of thousands, of kilometers from a space-based telescope far removed from gravitational bodies. The occulter would be commanded to interpose itself directly between the telescope and a target star in order to block the light from the star. This would enhance the ability of the telescope to detect any faint companions (i.e., planets) that are in orbit about the target star. The principle is identical to blocking the sunlight from one's eyes with a hand in order to observe something close to the Sun - an easy task to perform. The challenge comes when trying to command a telescope and an occulter to do the same thing, but with a distant and fainter star. The idea of a free-flying occulter paired with a space telescope was first suggested in the early 1960s [2]. In the subsequent 40 years, many ideas and designs have been put forth [3-14]. This paper is not meant to discuss the general occulter designs, but rather the requirements that must be met in order to actually operate a space telescope/occulter pair [15].

The main goal of a five year mission would be to detect and study extrasolar planets about the stars within 15 parsecs of the Sun - approximately 300 targets. Simulations show that an apodized telescope with a free-flying occulter could easily detect a Jupiter-like planet about a star at 10 parsecs [16]. The telescope and occulter spacecraft fly in formation in the vicinity of the Sun-Earth L2 point, separated by tens of thousands of kilometers. The occulter blocks stellar light from entering the telescope. When occulter science is completed, the occulter transits to the next 
target station, then the cycle repeats. Between occulter targets, the telescope will be used for other science.

\section{System \& Mission Requirements for 2 Spacecraft Operations}

The occulter mission design is driven by many different constraints, and there are trade-offs involved in trying to meet those constraints. These include cost (build two spacecraft, launch, and operations), data transfer (between Earth and occulter and between telescope and occulter), occulter observation rate, etc. The goal is to minimize constraints while maximizing mission science.

\section{Mission Design - Location, Location, Location:}

The critical mission constraint is alignment of the occulter on the line of sight from the telescope to the target star, while at a large distance from the telescope. The target-telescope line of sight (TTLOS) moves because the telescope moves in its orbit, and so the TTLOS point for the occulter moves. The telescope/occulter pair must be placed in orbits where the differential motion between the TTLOS point and the occulter's orbital position can be minimized (see [15] and further sections in this paper). This constraint can be satisfied by solar orbits with semi-major axis of $1 \mathrm{AU}$ or larger.[15] Communication requirements (see below) dictate a position relatively close

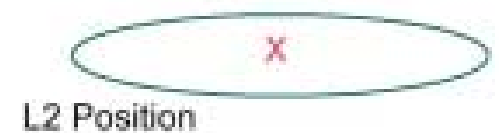

L2 Position

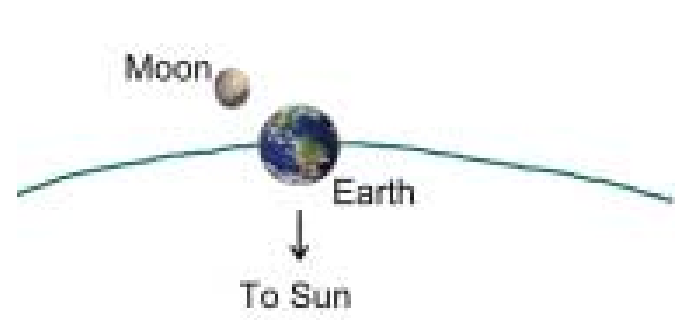

to Earth. Orbits near the Sun-Earth L2 point or orbits which trail or lead the Earth can satisfy both communication needs and the alignment constraint. However, Earth trailing/leading orbits would impose an Earth/Moon avoidance zone on the science scheduling system. L2 imposes no such avoidance zone, because the Earth and Moon will be within the solar avoidance zone (compare the illustration of the $\mathrm{L} 2$ region in Figure 1 with Figure 3). The rest of this paper assumes the telescope and occulter are located in orbits near the Sun-Earth L2 point.

Figure 1: The L2 region relative to the Earth-Moon system

\section{Communication:}

The occulter movement and alignment events with the telescope need to be autonomous, as the pair will be out of ground communication for nearly two thirds of each day. For the simplest operations model, we assume there will only be one prime ground station communicating with the telescope/occulter pair. The period of time that the ground station can communicate with the occulter and telescope would nominally be a 10-hour period each night, when the L2 region of the sky (anti-Sun as seen from Earth) would be above the horizon and visible. It is only during these 
times of contact that science data can be downlinked, the latest Observation Plan (OP) or events calendar uplinked, and commanding changes for the telescope and occulter sent. The ground station will not have any capability to communicate with the telescope/occulter pair while the L2 region is blocked by the Earth (i.e., when the ground station is on the daytime side of Earth) unless additional links are provided by other ground-to-space networks.

Under normal operations, communications from the ground station to the occulter will typically be sent to the telescope, which will relay it to the occulter. The occulter will likewise send its telemetry to the ground station via the telescope. However, the occulter must have the capability of being able to communicate (receive and transmit) directly with the ground station whenever an off-nominal situation occurs and during transit to the next target station.

\section{Data Transfer Rates:}

The space telescope will have a complement of science instruments onboard for a variety of observing modes. The storage capacity onboard the telescope needs to be fairly substantial, and we estimate these requirements here. When doing occultation observations with the occulter, it is estimated that the telescope will take on the order of 100 science exposures per day (more if the exposures are short).

Multiple alignment exposures may be required to realign the occulter onto the TTLOS, as the occulter may very well have drifted off. Assuming a minimum of 4 realignment images per science exposure (in order to account for drift of the two spacecraft), approximately 500 exposures (science and drift realignment) per day may be obtained, resulting in approximately $11 \mathrm{~Gb}$ of data per day. In order to accommodate engineering telemetry data and retransmissions, 18Gb of storage may be needed. With a downlink rate of approximately $1 \mathrm{Megabit} / \mathrm{second}$, approximately 5 hours of downlink time from the telescope is needed, which is a significant amount of the visibility window with the telescope/occulter pair. The remaining time can be used for any uplink transmissions to the telescope and/or occulter. However, uplink volume will be comparatively small, $\sim 10 \%$ of the download, and would not amount to a significant fraction of the available time. Simultaneous upload/download is possible as long as two distinct bands (e.g., S-Band and X-Band) are utilized.

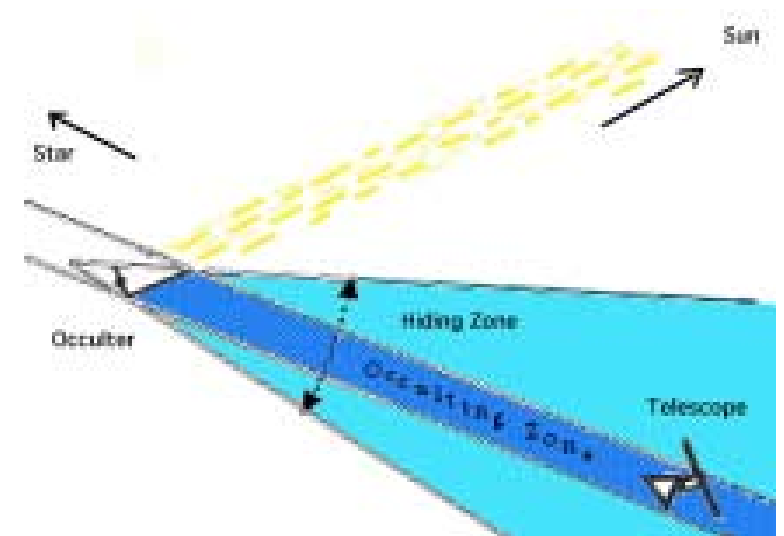

Figure 2: Target-Telescope-Line-Of-Sight with occulter 


\section{Occulter Beacons:}

In addition to typical science instrumentation and other components on the space telescope (enabling it to operate and observe independent of the occulter), the telescope will also have a sensor suite to locate the occulter's navigational and alignment beacons for positional referencing and alignment. If the beacons have frequencies far outside the observational bandpass of the science observations, then they will be able to operate continuously (eliminating the possibility that one or more might not turn on after being turned off), allowing the telescope to constantly track the occulter as a low-level monitoring activity. The beacons, detectable over distances of thousands of kilometers, would not need to be more powerful than a few Watts (with a beamwidth of 5 degrees).

\section{Spacecraft Operations Domains}

Occulter operations are partly dependent on architecture. The main functional components are power, propulsion (occulter only), attitude control, and formation control. Both occulter and telescope will be solar powered. The occulter will use Solar-Electric Propulsion (SEP) units for movement. The main engine, a high-thrust SEP unit, will be used to propel the occulter between target stations. Several low-thrust SEP units will be used for Attitude and Translation Control (A\&TC) to maintain occulter alignment on the TTLOS. Xenon gas is currently the most likely propellant for both the main engine and the A\&TC engines. Reaction wheels will also be used for attitude control. Gyros and star trackers will be used for rate and attitude sensing, respectively. Formation Flying control will be enabled by beacons on the occulter and sensor systems on the telescope to detect and locate the occulter beacons.

\section{Guidance, Navigation, and Control:}

The attitude of the occulter (its orientation in space) is sensed and maintained through a guidance, navigation, and control (GN\&C) subsystem. The GN\&C subsystem of the occulter (and of the telescope) will be of paramount importance to meet formation flying objectives. Guidance is the process of defining the spacecraft's desired motion in time, either in an absolute or relative reference frame. Navigation is the process of measuring and estimating the vehicles' positions and/or orientations, while relative navigation is the determination of the relative states between the telescope and the occulter. Finally, control is the application of corrective forces and moments on the vehicle(s) when the vehicles are not on their desired absolute and/or relative trajectories, accounting for perturbations, disturbances, and unmodeled dynamics. The process of measuring and controlling the relative states of the vehicles will be denoted formation flying. The need for formation flying drives a GN\&C architecture that spans all vehicles in the formation. In this case, the occulter will have very limited computational capability so the telescope will host a centralized GN\&C subsystem for the formation (i.e., a formation flying subsystem, or FFS). The FFS will be 
responsible for measuring and maintaining desired relative geometry between the spacecraft and occulter, with most functions handled on the telescope spacecraft.

\section{GN\&C Drivers and Constraints:}

The space telescope's frame of reference is defined by the location of the Sun and a catalogue of guide stars. Once the telescope's state (position and attitude) relative to the frame of reference has been determined, planning the trajectory of the occulter from target to target becomes possible. The occulter will use the Sun, guide stars, and the space telescope for guidance, navigation, and control.

There will be stringent requirements on the occulter's motion relative to the telescope. During observations requiring the use of the occulter, the occulter will be confined in a ring-shaped region oriented perpendicular to the Sun/Anti-Sun line through the space telescope. This region is known as the Quadrature Ring (QR). The QR will typically be tens of thousands of kilometers in diameter (typical occulter-to-telescope ranges will be on the order of 10,000-20,000 kilometers, where the range is a function of distance to the target and size of the occulter). It is crucial that the occulter be in the QR as observing constraints require the normal to the occulter surface to be as perpendicular to the Sun/Anti-Sun direction as possible. This is primarily to reduce either direct light from the Sun entering the telescope's aperture, or of light reflecting off of the occulter, defeating any benefit the occulter would have provided in reducing the light from a distant star.

The QR is not a simple torus, but has some avoidance regions along cones of revolution around the Sun/Anti-Sun line (see Figure 3), potentially of different angles for the two different directions. Also, the inner and outer surfaces of the QR are bounded by optimum science goals.

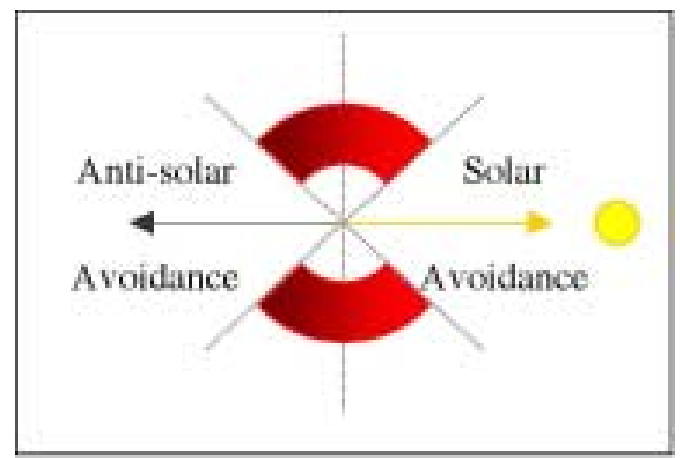

Figure 3: The Quadrature Ring

\section{Spacecraft Management:}

The primary requirements of the occulter mission are to achieve adequate formation control of the occulter craft and the space telescope such that the occulter remains on the TTLOS vector. The lifetime of the occulter mission depends upon the amount of propellant carried, the propulsion system, thrust and specific impulse, the distance between the telescope and occulter, the number of targets, and the rate of supported occulter observations. The occulter craft needs to be monitored for health and safety, fuel usage, acceleration, and positioning. Power management is 
part of nominal operations for the telescope, as there is essentially no difference between observing with or without the occulter. On the other hand, the occulter has two different power modes: transiting and observing. During observing mode, the occulter will have excess power that would have otherwise been used by the ion engine during transits.

Momentum management is dependent upon the design of the vehicles, and will affect the alignment of the vehicles. The Observing Plan will enforce no momentum dumps while science observations are in progress and will schedule momentum dumps while on the target station when necessary. Momentum must be dumped prior to observing sequences.

\section{Inter-spacecraft Operations}

Operation of a free-flying occulter requires maneuvering between stations, alignment on the TTLOS, and formation control. A key element of formation control is the process of maintaining the occulter on the TTLOS with sufficient accuracy to allow useful science observations.

When the occulter is not used for science, it will be in transit between target stations. The space telescope will not be idle during this time, but instead be used for non-occulter observations. During this period the telescope and occulter will maintain low-level contact for basic relative location information.

\section{Formation Maneuvering and Initialization:}

The FFS must be designed so that the occulter, upon orbital insertion or after moving thousands or tens of thousands of kilometers from a previous target station to the next, can be quickly brought into an initial alignment on the TTLOS. Fine alignment and formation flying with the telescope must then proceed. This will nominally be accomplished through telescope-occulter communications, and will require the telescope to be able to detect and store the location of the occulter with respect to the TTLOS. If the occulter begins to drift off the TTLOS, the telescope will compute the current and desired vectors and send this information to the occulter. The occulter will execute the re-alignment maneuvers. The following is an example of an alignment sequence [17]:

1. Occulter transits toward the new target station, but has not yet reached the field.

2. Occulter signals the telescope, triggering an interrupt flag.

3. Telescope takes reference images of the field.

4. Occulter arrives near the TTLOS (initial alignment).

5. Occulter articulates into observing configuration while still off the TTLOS.

6. While the occulter is off the TTLOS: 
a. Telescope beacon sensor is used to measure occulter location and velocity.

b. Telesope alignment imager is used to measure sun-glint.

c. Relative position uplinked to occulter.

7. Occulter translates into alignment along TTLOS.

8. Telescope alignment imager images occulter.

9. Alignment determined, compute state vector.

10. If velocity cannot be satisfactorily computed for, return to Step 6a.

11. Uplink the computed and desired occulter state vector from the telescope to the occulter.

12. Occulter computer determines and executes alignment maneuver sequence.

13. Telescope images target field to verify alignment.

14. If alignment does not pass check, return to Step 6a.

15. Proceed with science, checking alignment after each observation is taken.

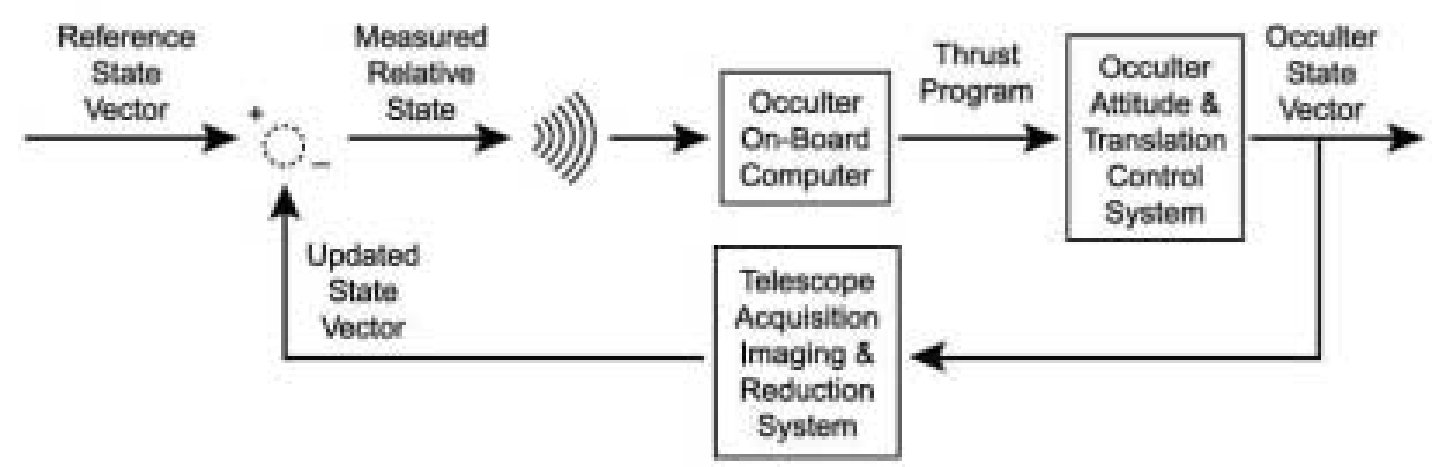

Figure 4: A representative control block diagram showing the basic feedback loop for telescope/occulter formation control. [15]

When the observing session for a given target is complete, the telescope will command the occulter to "stand down" from observing mode and proceed into transit mode. The occulter will then reorient and move to the next station. Upon reaching the next station, it communicates this to the telescope, and the alignment procedure begins. As contact with the ground station will be restricted, the entire alignment sequence must be autonomous.

\section{Science \& Mission Support}

There are two classifications of science observations that the telescope will execute: occulter observations and non-occulter observations. The non-occulter observations are general astronomical observations. Occulter observations would be those in which the telescope and occulter interact to observe a target. Direct detection and study of extrasolar planetary systems is the primary purpose of the occulter in this mission. However, non-occulter science may have up 
to $80 \%$ of the total telescope time. Observations of an occluded target may take from a few days to upwards of a week to complete.

\section{Occulter Science Trigger:}

Because of inherent uncertainties in the event timing, the Observation Plan will be an eventdriven calendar that the space telescope executes sequentially. The telescope would check for an interrupt flag after each observation. The occulter would trigger an interrupt flag on the telescope when it is in position to begin alignment. Once an interrupt flag has been triggered, the telescope would complete its current observation, then transition to the alignment sequence.

\section{Operations and Commanding Teams:}

It is assumed that there would only be one transmitter/receiver ground station. It is envisioned that two shifts of personnel will be needed to maintain and support the telescope/occulter mission: an Operations Shift during the night hours when the L2 position is visible and when direct communication with the telescope/occulter pair is possible, and a Commanding, Planning, and Analysis shift during the daytime hours when the $\mathrm{L} 2$ is below the horizon.

The Operations Team will be tasked with downloading the science data from the telescope, downloading the engineering telemetry data from both the telescope and occulter, uploading new Observation Plans to the telescope as needed, and have direct control of the telescope and occulter if absolutely required. In addition to this the Operations Team would be involved with the science data processing and archiving.

The Commanding Team is responsible for generating the command loads, the Observation Plans, and responding to events and problems uncovered during the night shift. The Commanding Team might also be involved with the data processing, and definitely would be involved with the data analysis in some fashion in order to refine future Observation Plans.

\section{Science Planning \& Scheduling:}

In order to generate Observation Plans, three unique software tools are needed for scheduling the telescope/occulter activities. These are a Visibility Predictor (VP), an Accessibility Predictor (AP), and a Sequence Planner (SP). All three of these tools will be necessary when planning target-to-target sequencing in order to maximize the use of the occulter. The VP, given any date, will provide target visibility for the occulter. The AP will provide fuel estimates and transit times on a per target basis. 
The most complex of the three tools would be the SP, which assumes a given starting position for the occulter. The SP will determine if the occulter can stay longer on the current target and how that may affect a subsequent target. The SP should be able to generate, rank, and display different target sequencing scenarios.

Over the course of 6 months the entire sky will be visible to the telescope/occulter pair in the QR, although the entire sky will not be instantaneously visible. Also, it takes the occulter time to move from one target station to another. The Observation Plan will need to take these constraints into account.

Due to the length of on-target observation time, there will be the opportunity to provide feedback in order to modify the Observation Plans. It may be necessary to update and/or modify the Observation Plan on timescales of a few days.

If there are any changes made to the Observation Plan, the Commanding Team must look into downstream impacts to target visits. If the time on target is lengthened, the tools outlined earlier will help to determine how to get the best efficiency. It may not be possible to visit the next target, or it may be necessary to cut a later target from the list. In order to facilitate the Commanding Team's work, they may generate multiple contingency Observation Plans.

\section{Summary}

We have presented a brief discussion on the operational requirements for a two spacecraft mission, a free flying occulter and space telescope. Except during maneuvers to maintain the formation configuration for occulter science, the occulter craft must be autonomous from the space telescope with propulsion, navigation, and communication systems. The telescope needs to have the ability to communicate with and command the occulter craft. The operation and commanding teams were outlined from experience of the authors with the Hubble Space Telescope. Navigation, communication, and commanding (decision making algorithms, a.k.a. artificial intelligence) systems need to be developed to support the proposed mission, but are not unexpected for a mission to the Sun-Earth L2 position.

\section{Acknowledgements}

Support for this work has been provided by Computer Sciences Corporation, Laurel, MD and by the Space Telescope Science Institute which is operated by the Association of Universities for Research in Astronomy, Inc., under NASA contract NAS 5-26555. We would like to thank Wayne Kinzel for input on limitations and requirements for spacecraft communications. 


\section{References}

[1] The UMBRAS website: http://www.umbras.org/

[2] L. Spitzer, American Scientist 50, p. 473, (1962)

[3] G. Woodcock, Phase I Technical Report NASA-CR-141856 (N75-24802), Contract NAS914323, (1975)

[4] C. Marchal, Acta Astronautica, Vol. 12, No. 3, p. 195, (1985)

[5] C.J. Copi \& G.D. Starkman, Space Telescopes and Instruments V, P. Y. Bely and J. B.

Breckinridge, eds., Proc. of SPIE 3356, p. 608, (1998)

[6] C.J. Copi \& G.D. Starkman, ApJ. 532, p. 581, (1999)

[7] A. Schultz, et al., Infrared Spaceborne Remote Sensing VIII, M. Strojnik and B. Andersen, eds., Proc. of SPIE Vol. 3759, p. 49, (1999)

[8] A. Schultz, et al., Infrared Spaceborne Remote Sensing VII, M. Strojnik and B. Andersen, eds., Proc. of SPIE Vol. 4131, p. 132, (2000)

[9] I.J.E. Jordan, et al, NGST Science and Technology Expo., E.P. Smith and K.S. Long, eds., ASP Conf. Series, Vol. 207, p. 468, (1999)

[10] I.J.E. Jordan, et al, AIAA Space 2000 Conference, AIAA 2000-5230, on CD, (2000)

[11] I.J.E. Jordan, et al, BAAS, 33, p. 902, (2001)

[12] A. Schultz, et al., High-Contrast Imaging for Exo-Planet Detection, Proc of SPIE Vol. 4860, p 54, (2002)

[13] M Kochte, et al., BAAS, 33, p. 1367, (2001)

[14] M Kochte, et al., The Search for Other Worlds, 14th Annual October Astrophysics Conference in Maryland, in press, (2003)

[15] H. Hart, et al, 2000 International Conference on Applications of Photonic Technology, Proc of SPIE Vol. 4087, p. 993 (2000)

[16] A. Schultz, et al., Techniques and Instrumentation for Detection of Exoplanets, Proc. of SPIE 5170-26, p. 262, (2003)

[17] I.J.E. Jordan, term paper for Sensors and Sensor Systems, JHU/WSE 615.747, (2003) 